\title{
Effect of Acid-Hydrolyzed Thermoplastic Starch on the Mechanical, Thermal and Morphological Properties of Polyethylene Based Composites
}

\author{
Perla Mayari Trejo-Ramírez ${ }^{1}$, Ernesto Hernández-Hernández ${ }^{2,3,{ }^{*},}$ Javier Castro-Rosas ${ }^{1}$, \\ Silvia Guadalupe Solís-Rosales ${ }^{2}$, José Roberto Villagómez-Ibarra ${ }^{1}$ and \\ Carlos Alberto Gómez-Aldapa ${ }^{1, *}$
}

${ }^{1}$ Universidad Autónoma del Estado de Hidalgo. Ciudad del conocimiento, Área Académica de Químicas, ICBI-UAEH, Carretera Pachuca-Tulancingo Km 4.5, Col. Carboneras, Mineral de la Reforma, Hidalgo, C.P. 42184, Mexico

${ }^{2}$ Centro de Investigación en Química Aplicada, Boulevard Enrique Reyna No. 140, Saltillo, Coah C.P. 25253, Mexico

${ }^{3}$ Consejo Nacional de Ciencia y Tecnología, Av. Insurgentes Sur \# 1582, Col. Crédito Constructor, Del. Benito, Juárez C.P. 03940, Mexico

\begin{abstract}
The effect of the addition of hydrolyzed thermoplastic maize starch on the physico mechanical properties of low-density polyethylene (LDPE)-based composites was studied. Acid-hydrolyzed native starch was thermoplasticized using 15 and $30 \%$ glycerol in weight relative to starch, after which the LDPE/thermoplastic starch (TPS) composites were prepared at TPS concentrations of 10,25, and $50 \%$. According to the results of Raman spectroscopy, the appearance of a new band at $756 \mathrm{~cm}^{-1}$ was observed, and it was attributed to the hydrolysis process and associated with the C-C-O vibrational modes of the glycosidic bond. The addition of both native and polyethylene hydrolyzed TPS reduced the Young's modulus of the composites; but the reduction was greater for those containing native starch. Both the maximum stress and deformation decreased to a greater degree for the composites with hydrolyzed TPS. The composites containing TPS prepared with 15\% glycerol exhibited a higher Young's modulus compared to those with LDPE, although they exhibited fragile behavior. The degree of matrix crystallinity increased with the addition of TPS and showed the largest increase when TPS $50 \%$ hydrolyzed by weight was added, showing an increase of $35 \%$. It was observed that the size of the TPS particles, both native and hydrolyzed, increased in size as the concentration of TPS in the matrix increased. The size of the hydrolyzed TPS particles was greater than that of the native TPS particles, and in the case the of the hydrolyzed TPS particles, some exhibited an ellipsoidal and/or fibrillar morphology.
\end{abstract}

Keywords: Chemical modifications, LDPE, hydrolyzed thermoplastic starch.

\section{INTRODUCTION}

The inadequate containment of large volumes of synthetic plastics, once they have completed their useful lifespan, has caused serious environmental problems. Therefore, there is a growing interest in the development of biodegradable materials to replace synthetic plastics and/or to help minimize their environmental impact. Among the different biodegradable polymers, starch has been the most extensively studied, mainly due to its widespread availability and low cost. This polymer is composed of D-glucose polysaccharides (98-99\%) and consists of two polymers: amylose, a linear polymer formed by $D$ glucose units linked by $\alpha-(1-4)$ bonds, and amylopectin, a branched molecule formed by D-glucose units linked

*Address correspondence to these authors at the Universidad Autónoma del Estado de Hidalgo. Ciudad del conocimiento, Área Académica de Químicas, ICBI-UAEH, Carretera Pachuca-Tulancingo Km 4.5, Col. Carboneras, Mineral de la Reforma, Hidalgo, C.P. 42184, Mexico; Tel: 527717172000 ext. 2518 or 2201; E-mail: cgomeza@uaeh.edu.mx

Centro de Investigación en Química Aplicada, Boulevard Enrique Reyna No. 140, Saltillo, Coah C.P. 25253, Mexico;

E-mail: ernesto.hernandez@ciqa.edu.mx by $\alpha-(1-6)$ and $\alpha-(1-4)$ bonds in its linear section [1]. The ratio of these two polysaccharides varies according to the botanical origin of the starch. An example is waxy maize starch, which contains a concentration of amylose of 1 to $2 \%$. This concentration gives it a low degree of viscosity, which makes it less prone to retrogradation and allows its structure to be more easily modified via chemical methods [2].

For the starch to be manageable and able to be processed, it has to undergo a hydrothermal process called gelatinization, which occurs under conditions of humidity and temperature. When a plasticizing agent such as glycerol is used in addition to water, a viscous paste called thermoplastic starch (TPS) is obtained. The properties of TPS depend on the concentration of the plasticizing agent, the type of starch, and the amylose/amylopectin ratio $[3,4]$. The mechanical properties of TPS are inferior to those of synthetic polymers, and it is highly hydrophilic [5, 6]; as a result its applications remain limited, which is why it has been 
recommended that they be mixed with synthetic polymers to obtain partially biodegradable materials with useful properties for a range of applications [7, 8]. In this regard, polyolefins have been the primary matrices to which TPS has been added [9], and the final properties of the composites are directly dependent on the following characteristics: the morphology of the TPS in the matrix [10], the TPS concentration, the amount of plasticizer that the TPS contains, and the type of starch, among other aspects [10]. It has generally been reported that the addition of TPS causes a reduction in the tensile strength, Young's modulus and elongation at the break of composites [11, 12]. This behavior is essentially due to the incompatibility of the two phases as a result of their different polarities [13, 14]. To make the reduction of these properties less significant, some compatibilizing agents such as maleic anhydride have been used. When grafted onto a polyethylene matrix, these compatibilizing agents improve the properties compared to non-maleated composites. Another alternative that has been explored is chemically modifying the starch at its $\mathrm{OH}$ groups to change its functionality and grant it new properties such as a reduction in the degree of retrogradation and hydrophobic properties [15-17].

Starch hydrolysis is a random process that generates a blend of polymers with different molecular weights that can be composed of anything from glucose monomers to polymers the size of starch molecules, depending on the severity of the hydrolysis process. Hydrolyzed starch is colorless, does not crystallize [18], has a lower viscosity, and is more easily biodegradable [3]. These properties can improve the properties of composites based on synthetic polymers, thereby increasing their possible applications. There are no reported cases in the literature on the impact of using hydrolyzed starch on the physicochemical properties of synthetic polymerbased composites.

On the basis of the above, the main aim of the present work is to determine the effect of the addition of acid-hydrolyzed thermoplastic starch (TPSH) on the physicochemical properties of low-density polyethylene (LDPE)-based composites. The hydrolysis of the granular starch is performed using hydrochloric acid, after which it is thermoplasticized in a mixing chamber using glycerol as a plasticizing agent. LDPE/TPSH composites in different proportions are then prepared, and the mechanical, thermal, and morphological properties of these composites are determined.

\section{MATERIALS AND METHODS}

\subsection{Materials}

This study used Infra brand Waxy maize starch from the commercial distributor Ferzabeth that contains $98 \%$ amylopectin, hydrochloric acid $(\mathrm{HCl})$ with a purity of $36.5 \%$, J.T. Barker brand analytical grade glycerol, and low-density polyethylene (LDPE) 20020 X-L 23-70334 produced by Petróleos Mexicanos (PEMEX).

\subsection{Acid Hydrolysis of the Waxy Starch}

Acid hydrolysis was performed using the methodology described by Zambrano and Camargo [19], with some modifications as described: The waxy starch $\left(\mathrm{S}_{\mathrm{N}}\right)$ was mixed with $3.4 \%(\mathrm{~V} / \mathrm{V}) \mathrm{HCl}$ in a $1: 5$ ratio, and the mixture was stirred with a magnetic stirrer for $4 \mathrm{~h}$ at a temperature of $50^{\circ} \mathrm{C}$. It was then neutralized with $20 \% \mathrm{NaOH}$, washed, centrifuged, dried, and stored for subsequent use.

\subsection{Thermoplasticization of the Waxy Starch}

Mixtures of granular starch with 15 and $30 \%$ glycerol by weight were prepared by adding water at $18 \%$ base weight to the starch, and the mixtures were left to stand for $24 \mathrm{~h}$. These starch/water/glycerol mixtures where thermoplasticized in a Brabender ATR Plasti Corder S/N K07-17A interior Mixer (Germany) at a speed of $60 \mathrm{rpm}$ for $20 \mathrm{~min}$ and at a temperature of $100{ }^{\circ} \mathrm{C}$, using CAM-type rotors for the process. The labels for the various plasticized starches are given in Table 1.

Table 1: Experimental Design to Obtain the Thermoplastic Starches (TPS)

\begin{tabular}{|c|c|c|}
\hline Type of starch & Glycerol (\%) & Blend \\
\hline \hline \multirow{2}{*}{$\mathrm{S}_{\mathrm{N}}$} & 15 & TPS $_{\mathrm{SN} 15}$ \\
\cline { 2 - 3 } & 30 & TPS $_{\mathrm{SN} 30}$ \\
\hline \multirow{2}{*}{$\mathrm{S}_{\mathrm{H}}$} & 15 & TPS $_{\mathrm{SH} 15}$ \\
\cline { 2 - 3 } & 30 & TPS $_{\mathrm{SH} 30}$ \\
\hline
\end{tabular}

$\mathrm{S}_{\mathrm{N}}$ : Native starch; $\mathrm{S}_{\mathrm{H}}$ : Hydrolyzed starch; TPS: thermoplastic starch; 15 or 30 (\%) glycerol used for thermoplasticization.

\subsection{Preparation of the LDPE/TPS Composites}

The LDPE/TPS composites were prepared in the melted state in the same chamber in which the starch was thermoplasticized. The composites were heated at $160{ }^{\circ} \mathrm{C}$ at $60 \mathrm{rpm}$ for $10 \mathrm{~min}$. First, the polyethylene pellets were added, and after $3 \mathrm{~min}$, when the polymer was fully melted, the TPS was added. The torque of the 
Table 2: LDPE/TPS, Low-Density Polyethylene (LDPE) and Thermoplastic Starch (TPS) Formulations

\begin{tabular}{|c|c|c|c|}
\hline \multicolumn{2}{|c|}{ TPS (\%) } & LDPE (\%) & Composites \\
\hline \multicolumn{2}{|c|}{-} & 100 & LDPE \\
\hline TPS $_{\mathrm{SN} 15}$ & 25 & 75 & $\mathrm{~A}(75 / 25)_{\mathrm{N} 15}$ \\
\hline \multirow[t]{3}{*}{ TPS $_{\mathrm{SN} 30}$} & 10 & 90 & $\mathrm{~B}(90 / 10)_{\mathrm{N} 30}$ \\
\hline & 25 & 75 & $\mathrm{C}(75 / 25)_{\mathrm{N} 30}$ \\
\hline & 50 & 50 & $\mathrm{D}(50 / 50)_{\mathrm{N} 30}$ \\
\hline TPS $_{\mathrm{SH} 15}$ & 25 & 75 & $E(75 / 25)_{H 15}$ \\
\hline \multirow[t]{3}{*}{ TPS $_{\mathrm{SH} 30}$} & 10 & 90 & $\mathrm{~F} \mathrm{90/10)})_{\mathrm{H} 30}$ \\
\hline & 25 & 75 & $\mathrm{G}(75 / 25)_{\mathrm{H} 30}$ \\
\hline & 50 & 50 & $\mathrm{H}(50 / 50)_{\mathrm{H} 30}$ \\
\hline
\end{tabular}

LDPE: low-density polyethylene; Composites: $A(75 / 25)_{\mathrm{N} 15}, \mathrm{~B}(90 / 10)_{\mathrm{N} 30}, \mathrm{C}(75 / 25)_{\mathrm{N} 30}, \mathrm{D}(50 / 50)_{\mathrm{N} 30}, \mathrm{E}(75 / 25)_{\mathrm{H} 15,} \mathrm{~F}(90 / 10)_{\mathrm{H} 30}, \mathrm{G}(75 / 25)_{\mathrm{H} 30}$, and $\mathrm{H}(50 / 50)_{\mathrm{H} 30}$; weight ratioLDPE/TPS letter A (75/25), B (90/10), C (75/25), D (50/50), E (75/25), F (90/10),G (75/25), and H (50/50); subscript N: native starch; subscript H: hydrolyzed starch; number 15 or 30 : percentage of glycerol in the TPS

composites was measured throughout the preparation using the Brabender mixer program software (Fusion Behaviour, Germany) version 3.2.29. The prepared formulations of the LDPE/TPS composites and their assigned names are given in Table 2 . In short, they can be interpreted as follows: A $(75 / 25)_{\mathrm{N} 15}$. The letter $A$ refers to the LDPE/TPS composite, 75/25 refers to the ratio by weight of LDPE/TPS, $\mathrm{N}$ is native starch, and the number 15 refers to the glycerol concentration in the TPS. The composites labeled with the subscript $\mathrm{H}$ are those that contain hydrolyzed starch.

\subsection{Characterization of the LDPE/TPS Composites}

The waxy and hydrolyzed starches were characterized via Raman spectroscopy using a Horiba brand micro-Raman model XploRA (Germany). The analysis was performed in the 1000 to $400 \mathrm{~cm}^{-1}$ frequency range with a $785 \mathrm{~nm}$ excitation laser under ambient conditions.

The LDPE/TPS composites obtained from the melted state were compressed into 15 per $15 \mathrm{~cm}$ plates with a thickness of $1 \mathrm{~mm}$ in a press at a temperature of $160{ }^{\circ} \mathrm{C}$ and a pressure of 25 tons. From these, type 1 test specimens were obtained according to the specifications established in the ASTM-D638-10 standard. Before being analyzed, the samples were conditioned at $50 \%$ relative humidity for $40 \mathrm{~h}$. Finally, the test specimens were analyzed in a Universal United CEF-80 machine (model 100-KNE) using a $500 \mathrm{~N}$ cell at a speed of $50.8 \mathrm{~mm} / \mathrm{min}$. A total of 5 measurements were taken for each sample.

The morphological properties of the composites were determined via scanning electron microscopy with a TOPCON brand microscope model SM-510 (Japan).
The samples were analyzed at a range of different magnifications and in various fields to obtain comprehensive information on the composites. A working distance of $18 \mathrm{~mm}$ was used. The samples were freeze-fractured and coated with a gold/palladium alloy to avoid surface charging during the analysis.

The thermal properties of the samples were determined using a TA Instruments (USA) brand differential scanning calorimeter (DSC) Discovery series model, and a heating-cooling cycle was used to erase the thermal history of the blends in the 30-200-30 -C temperature interval. The analysis was performed with a nitrogen flow of $50 \mathrm{~mL} / \mathrm{min}$, using a heating and cooling speed of $10^{\circ} \mathrm{C} / \mathrm{min}$. The value for the enthalpy of fusion was taken from the second heating run to obtain a more precise value with this methodology. The value of $\Delta \mathrm{H}$ from the second heating run was used to calculate the degree of crystallinity of the composites according to Equation 1:

$X c=\frac{\Delta H_{f}^{*}}{\Delta H_{f}(1-\varnothing)} \times 100$

Equation 1

where $\Delta \mathrm{H}_{f^{*}}$ is the enthalpy of fusion of the composite obtained by DSC; $\Delta \mathrm{H}_{f^{*}}$ is the enthalpy of PE with a crystallinity of $100 \%$, whose reported value is $295.8 \mathrm{~J} / \mathrm{g}$ [20]; and $\varnothing$ is the fraction by weight of the dispersed phase of the composite.

\section{RESULTS}

\subsection{Starch Hydrolysis Studied by Raman Spectroscopy}

Figure 1 shows the Raman spectra of the native $\left(\mathrm{S}_{\mathrm{N}}\right)$ and hydrolyzed $\left(\mathrm{S}_{\mathrm{H}}\right)$ starches. There is a good 
overall correspondence of the spectra to those reported by Almeida et al. [21]. In the $\mathrm{S}_{\mathrm{N}}$ spectrum, the assignment of the observed bands is as follows. The intense band between 2800 and $3000 \mathrm{~cm}^{-1}$ corresponds to the $\mathrm{C}-\mathrm{H}$ stretching vibration. The band at $1457 \mathrm{~cm}^{-1}$ is attributed to the bending mode of the C$\mathrm{H}, \mathrm{CH}_{2}$, and $\mathrm{C}-\mathrm{O}-\mathrm{H}$ groups. The signal at $1397 \mathrm{~cm}^{-1}$ is due to the bending vibration of the $\mathrm{C}-\mathrm{C}-\mathrm{H}$ group, whereas the signal at $1377 \mathrm{~cm}^{-1}$ is associated with the $\mathrm{C}-\mathrm{O}-\mathrm{H}$ bending vibration. The intense signal at 1337 $\mathrm{cm}^{-1}$ is due to a combination of the $\mathrm{C}-\mathrm{O}$ stretching vibration and the bending mode of the $\mathrm{C}-\mathrm{O}-\mathrm{H}$ group. The medium-intensity band at $1258 \mathrm{~cm}^{-1}$ is attributed to the bending modes of the $\mathrm{C}-\mathrm{C}-\mathrm{H}, \mathrm{O}-\mathrm{C}-\mathrm{H}$, and $\mathrm{C}-\mathrm{O}-\mathrm{H}$ groups. A low-intensity signal is observed at $1206 \mathrm{~cm}^{-1}$, which corresponds to the $\mathrm{C}-\mathrm{C}$ and $\mathrm{C}-\mathrm{O}$ stretching vibrations. The intense band situated at $1124 \mathrm{~cm}^{-1}$ corresponds to the stretching vibrations of the $\mathrm{C}-\mathrm{O}$ and $\mathrm{C}-\mathrm{C}$ groups and the bending mode of the $\mathrm{C}-\mathrm{O}-\mathrm{H}$ group. The bands between $810-975 \mathrm{~cm}^{-1}$ correspond to the C$C$ stretching vibrations and the signals below $800 \mathrm{~cm}^{-1}$ correspond to the skeletal vibrational mode of the glucopyranose ring $[22,23]$.

The Raman spectra of the hydrolyzed samples exhibit a decrease in the intensity of most of the bands compared to the native starch. However the appearance of a new band at $756 \mathrm{~cm}^{-1}$ was observed and was attributed to the hydrolysis process and associated with the C-C-O vibrational modes of the glycosidic bond. There are also changes in some of the bands, for example the band associated with the C-O stretching vibration, which is situated at approximately
$1334 \mathrm{~cm}^{-1}$, shows a shift of $3 \mathrm{~cm}^{-1}$ to a lower frequency. This shift may occur because there is less strain on this group as a result of a decrease in hydrogen bonding interactions, in addition to the process of hydrolysis itself. Another low-intensity band that appears as a product of hydrolysis is the band that appears at approximately $1302 \mathrm{~cm}^{-1}$, which may be associated with a bending vibration of the C-O group. This band is not observed in the $S_{N}$ spectrum, and its appearance may be the result of the hydrolysis process. The intensity of the band at $1206 \mathrm{~cm}^{-1}$ shown a significant decrease after hydrolysis has occurred.

As indicated above, an almost overall decrease in the intensity of the $S_{H}$ spectrum, compared to that of $S_{N}$ can be observed. However, this decrease is particularly noticeable in the alpha $\mathrm{C}-\mathrm{C}$ bands contained within the $920-960 \mathrm{~cm}^{-1}$ range. These bands are of great importance, according to Phillips et al. [24], who report a decrease in the intensity of these bands as the amylose concentration increases. This finding is supported by the fact that, during the process of hydrolysis, the bonds of the waxy starch molecules break, mainly those situated in the amorphous zones where the highest number of the $\alpha-(1-6)$ bonds are found. This result is consistent with the type of starch used, which contains a greater number of amylopectin molecules, that, when hydrolyzed at the $\alpha-(1-6)$ bonds, may release linear chains with a low degree of polymerization. This release is reflected in the decrease in intensity of the bands, as noted by the previously cited authors.

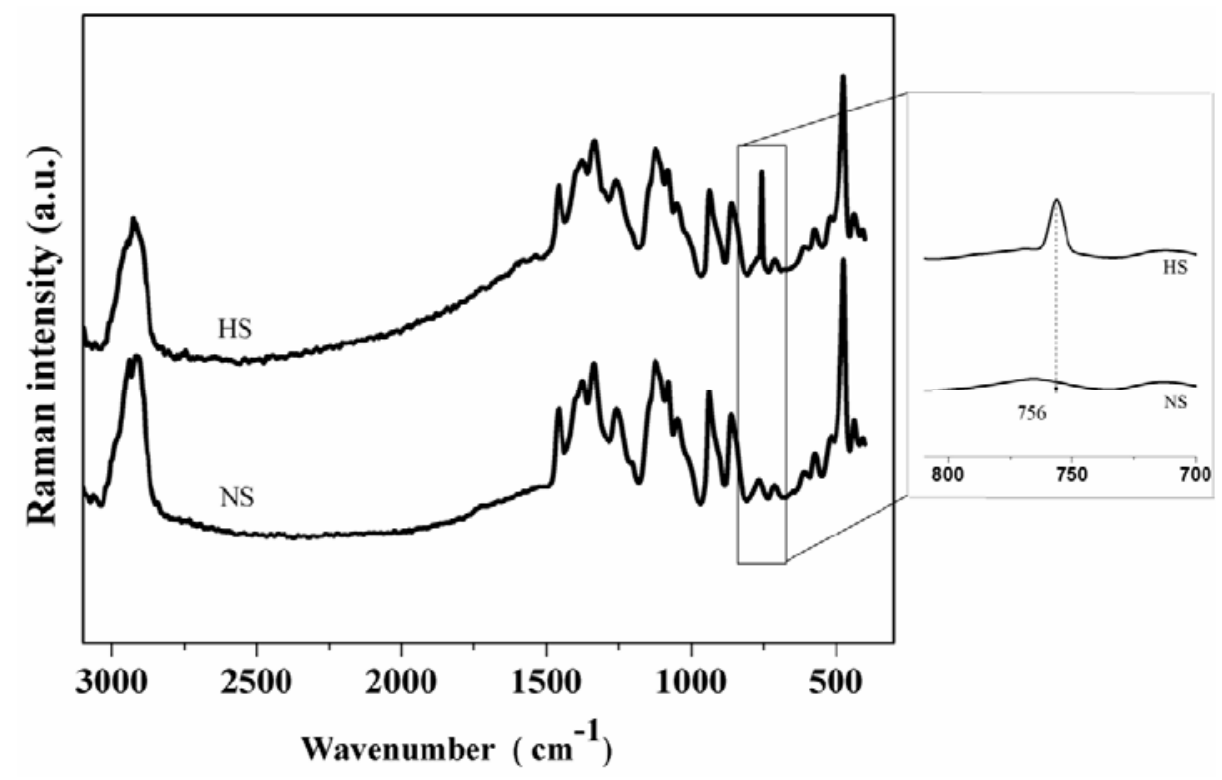

Figure 1: Raman spectra of the native (NS) and hydrolyzed (HS) starches. 


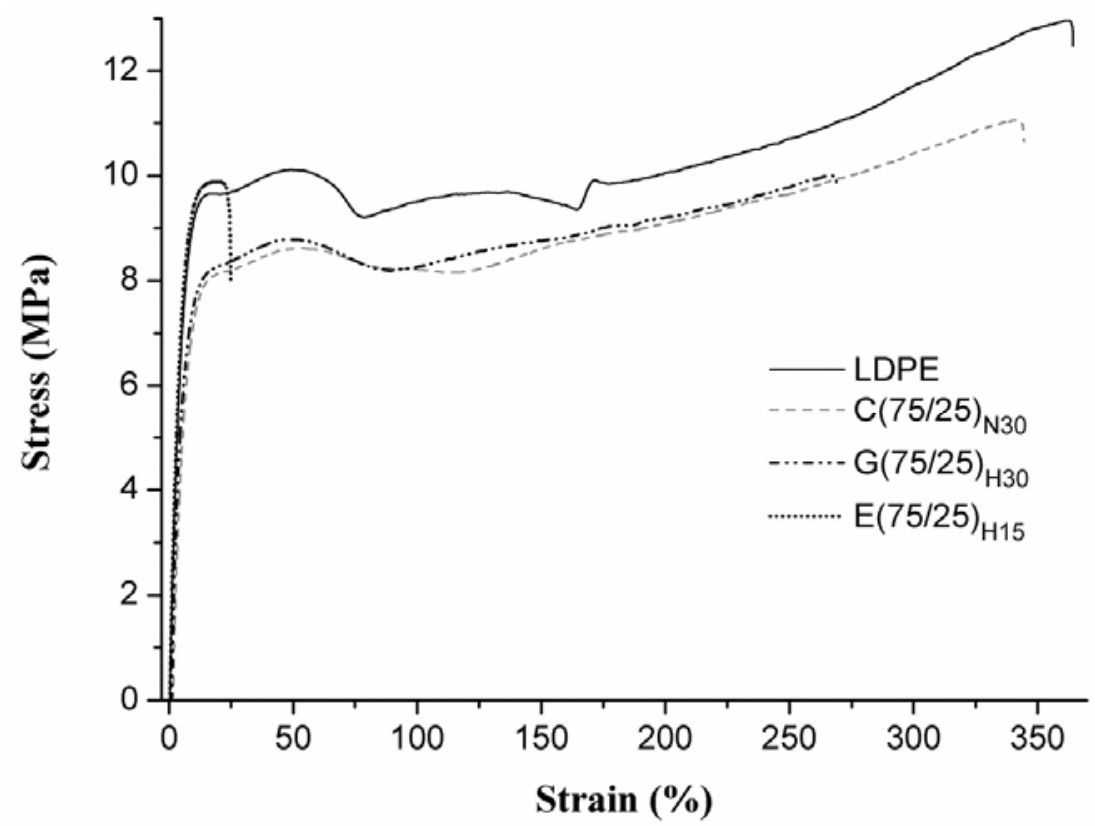

Figure 2: Stress-strain diagrams of LDPE and the LDPE/TPS compounds, native and hydrolyzed (75/25), with 30\% glycerol (C, $\mathrm{G})$ and hydrolyzed TPS with $15 \%$ glycerol (E).

Although it cannot be observed clearly in Figure 1, the band associated with amylopectin is situated at approximately $1637 \mathrm{~cm}^{-1}$, in agreement with the values in the literature [24]. The band at $1657 \mathrm{~cm}^{-1}$ attributed to amylose is also missing because waxy starch was used in this study. The absence of both of these bands can be attributed to the wavelength used $(785 \mathrm{~nm})$ and the conditions under which the analysis with this technique was performed, among other reasons.

\subsection{Stress-Strain Properties of the LDPE-Starch Composites}

The stress-strain diagrams of LDPE and the LDPE/TPS composites in a ratio of $75 / 25$, with native and hydrolyzed starch, respectively, are shown in Figure 2. The composites containing native and hydrolyzed TPS with 30\% glycerol exhibit stress-strain curves similar to that of LDPE, although both the stress and the strain of the composites are lower than those obtained with pure LDPE.

However, when the glycerol content used in the thermo plasticization of the starch is $15 \%$, the resulting stress-strain diagram has the shape of that of a material of low ductility, although the maximum stress of the material is similar to that of LDPE. This finding clearly demonstrates the effect of the glycerol concentration on the properties of the TPS, which is fragile with a glycerol content of $15 \%$ and ductile with a glycerol content of $30 \%$.
In order to assessment the effect of the native and hydrolyzed starches on the mechanical properties of the composites, in Figure $\mathbf{3}$ is shown the Young's modulus of composites LDPE/TPS containing of $30 \%$ glycerol. In general, it can be observed that the Young's modulus decreases as the TPS concentration increases and the same trend was observed in the both native and hydrolyzed starches. The Young's modulus decreases by up to approximately $32 \%$ when the TPS concentration of the composite is $50 \%$. The value of the Young's modulus in the LDPE/TPS (75/25) composites with hydrolyzed starch is higher compared to the composites with native starch. This improvement in the Young's modulus for the composites containing hydrolyzed starch may be related to the morphology (a decrease in the size of the dispersed phase) of the composite during its preparation because the hydrolyzed starch exhibits a lower viscosity compared to native starch. The decrease in Young's modulus with an increase in TPS concentration can be explained by the fact that this material is less rigid than the matrix, resulting in a corresponding decrease in this property in the composite. This result corresponds to what has previously been reported in the literature [25].

With regard to the maximum stress $\left(\sigma_{\max }\right)$ that the composites are able to endure as a function of the TPS concentration, Figure $\mathbf{4}$ shows a graph that demonstrates this trend. The LDPE matrix exhibits the highest maximum stress of $12.2 \mathrm{MPa}$ in comparison to the various LDPE/TPS composites that contain $30 \%$ 


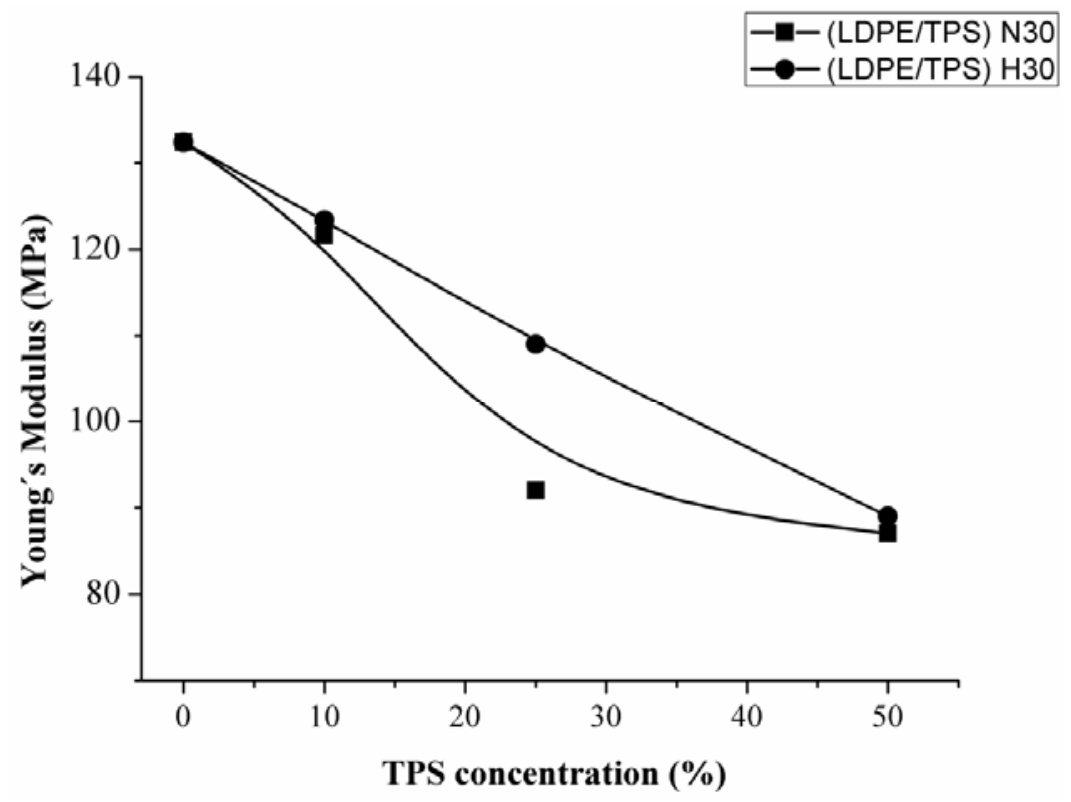

Figure 3: Young's modulus (E) of LDPE and the native and hydrolyzed LDPE/TPS composites as a function of the concentration of TPS containing 30\% glycerol. Native LDPE/TPS: $\mathbf{B}(\mathbf{9 0} / \mathbf{1 0})_{\mathrm{N} 30}, \mathbf{C}(\mathbf{7 5} / \mathbf{2 5})_{\mathrm{N} 30}$, and $\mathbf{D}(\mathbf{5 0 / 5 0})_{\mathrm{N} 30}$; and hydrolyzed LDPE/TPS: $\mathbf{F}(90 / 10)_{\mathrm{H} 30}, \mathbf{G}(75 / 25)_{\mathrm{H} 30}$, and $\mathbf{H}(50 / 50)_{\mathrm{H} 30}$.

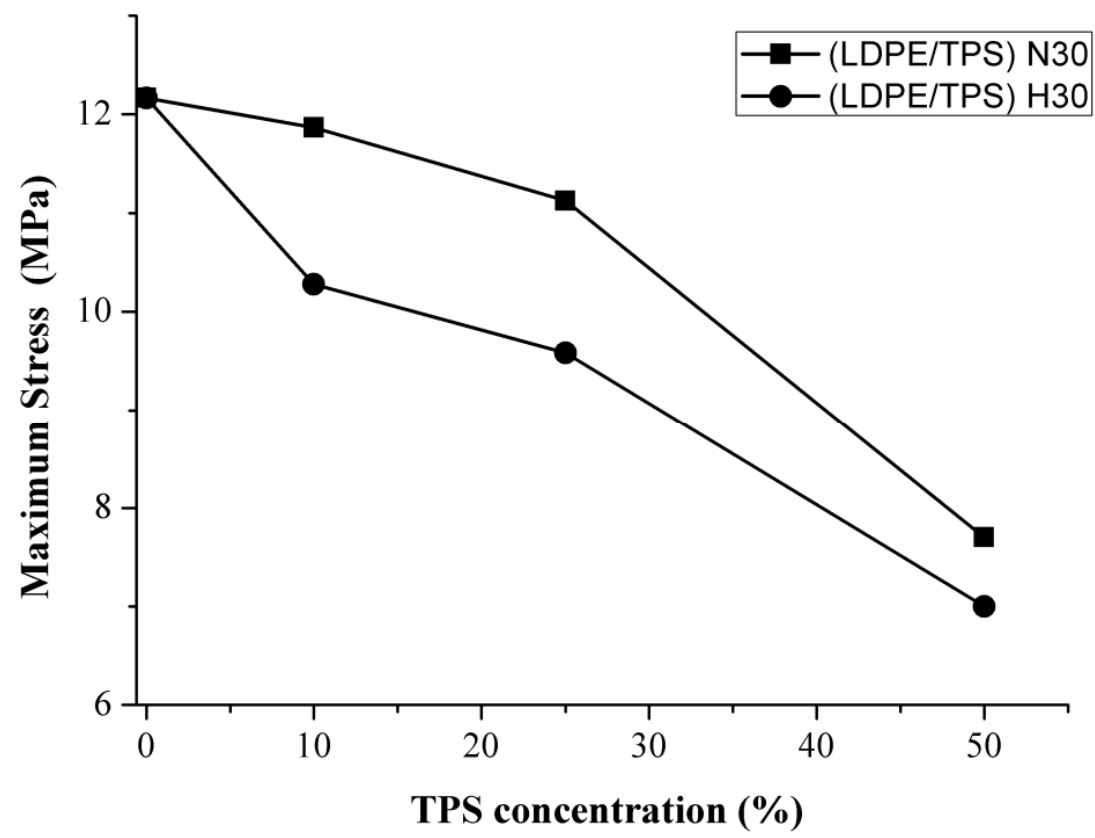

Figure 4: Maximum Stress ( $\sigma_{\max }$ ) of LDPE and the native and hydrolyzed LDPE/TPS composites at different concentrations of TPS containing $30 \%$ glycerol. Native LDPE/TPS: $\mathbf{B}(\mathbf{9 0} / \mathbf{1 0})_{\mathrm{N} 30}, \mathbf{C}(\mathbf{7 5 / 2 5})_{\mathrm{N} 30}$, and $\mathbf{D}(\mathbf{5 0 / 5 0})_{\mathrm{N} 30}$; and hydrolyzed LDPE/TPS: $F(90 / 10)_{\mathrm{H} 30}, G(75 / 25)_{\mathrm{H} 30}$, and $\mathbf{H}(50 / 50)_{\mathrm{H} 30}$.

glycerol relative to starch. As was the case for Young's modulus, the maximum stress of the composites decreases with the increase in TPS concentration. In the case of the composites that contain hydrolyzed starch, these exhibit a lower value than those that contain native starch. This effect can be attributed to the fact that the hydrolyzed starch deforms more easily during the deformation of the composite, in addition to the morphological difference of the dispersed phase, resulting in reduced stress transfer. The maximum stress of the LDPE/TPS 50/50 composites is reduced by up to $42 \%$ when the TPS is hydrolyzed and by up to $36 \%$ when the TPS comes from the native starch. Park et al. [26] have previously noted that the typical tendency of these types of blends is that both the increase in the TPS concentration of the composite and 


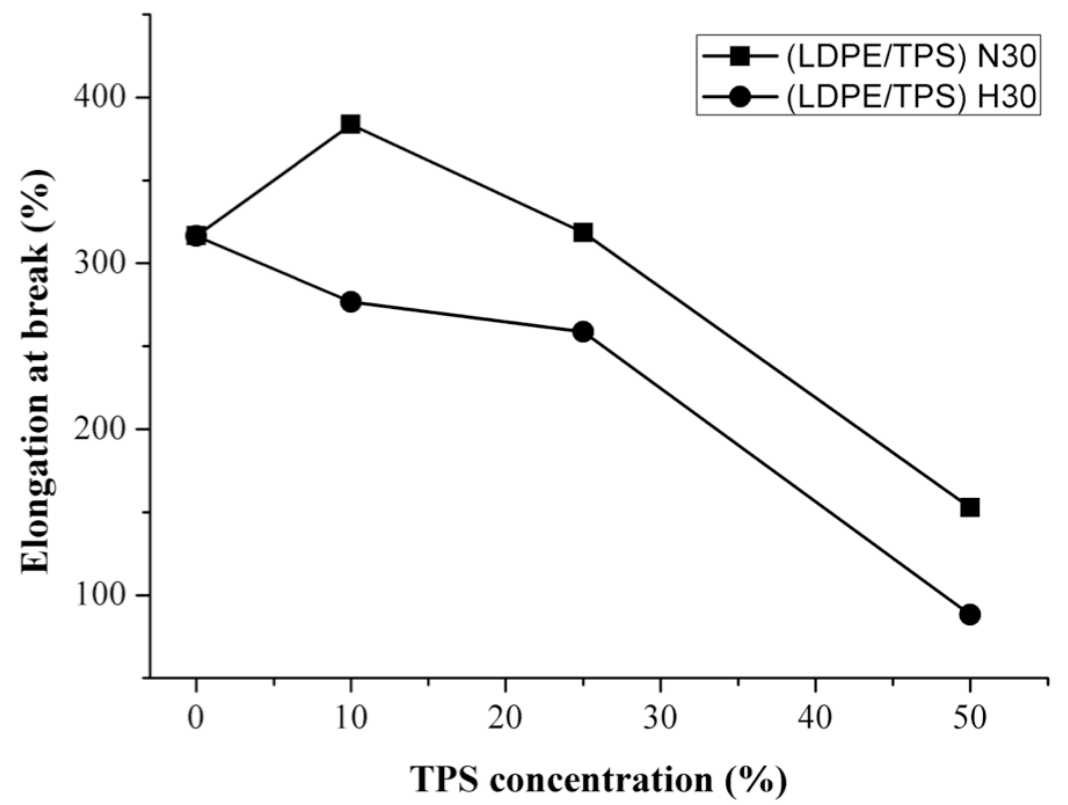

Figure 5: Elongation at break $\left(\varepsilon_{\mathrm{b}}\right)$ of LDPE and the LDPE/TPS composites at different concentrations of TPS containing $30 \%$ glycerol. Native LDPE/TPS: $\mathbf{B}(\mathbf{9 0} / \mathbf{1 0})_{\mathrm{N} 30}, \mathbf{C}(\mathbf{7 5 / 2 5})_{\mathrm{N} 30}$, and $\mathbf{D}(\mathbf{5 0 / 5 0})_{\mathrm{N} 30}$; and hydrolyzed LDPE/TPS: $\left.\left.\mathbf{F ( 9 0 / 1 0}\right)_{\mathrm{H} 30,} \mathbf{G ( 7 5 / 2 5}\right)_{\mathrm{H} 30}$, and $\mathrm{H}(\mathbf{5 0 / 5 0})_{\mathrm{H} 30}$.

the addition of glycerol contribute to a reduction in the maximum stress of the composites, which is in agreement with the findings of the present study.

The tendencies with regard to the elongation at break $(\varepsilon)$ of the LDPE/TPS composites that contain TPS originating from both native and hydrolyzed starch, with $30 \%$ glycerol, are shown in Figure 5. For the composites that contain TPS originating from hydrolyzed starch, in all cases, a reduction in the deformation with respect to the matrix is observed, although the reduction is not significant for TPS concentrations of 10 and $25 \%$. However, when the concentration is $50 \%$ by weight, the deformation is reduced by up to approximately $70 \%$. When the composites that contain TPS originate from native starch, the composites become more ductile at a concentration of $10 \%$ by weight of TPS, even more so than the matrix because the deformation increases by approximately $20 \%$. When the concentration of the native starch TPS is $25 \%$ by weight, the deformation is similar to that of the matrix, but when the concentration of the TPS is $50 \%$ by weight, a drastic reduction in the deformation of up to $52 \%$ occurs. The above results clearly indicate that the addition of hydrolyzed starch causes a greater reduction in the deformation of the composites relative to the composites that contain native TPS.

Each of the properties evaluated and extracted from the stress-strain diagrams for all of the prepared formulations are summarized in Table $\mathbf{3}$, highlighting the fact that in the case of the LDPE/TPS (75/25) composites containing TPS with a $15 \%$ glycerol content, a reduction in the deformation of up to 98 and $93 \%$ is observed for native and hydrolyzed TPS, respectively. This drastic decrease in the deformation indicates that a glycerol content of $15 \%$ is not sufficient to make the TPS flexible; indeed, it becomes brittle. A positive effect in the LDPE/TPS composites containing TPS with a glycerol content of $15 \%$ is observed in Young's modulus, which increases slightly, in the range of approximately $25 \%$. At this glycerol concentration, the TPS behaves as a typical filler in the matrix, as has been previously reported $[27,28]$.

\section{Thermal Properties of the LDPE/TPS Composites}

The thermal properties of the composites are given in Table 4. The data are obtained from the DSC thermograms of the second heating run, and based on these data, it can be observed that neither the fusion temperature nor the crystallization of the matrix is significantly affected by the presence of TPS. With regard to crystallinity, a slight increase with the addition of TPS is observed, in particular for TPS concentrations of 25 and $50 \%$. The increase in crystallinity with native and hydrolyzed TPS $(50 \%$ by weight in the matrix) is 26 and $35 \%$, respectively. The increase in crystallinity of the LDPE appears to have had no significant effect on Young's modulus, given that it is precisely the LDPE/TPS (50/50) composites 
Table 3: Summary of the Mechanical Properties of the Native and Hydrolyzed LDPE/TPS Composites

\begin{tabular}{|c|c|c|c|c|c|}
\hline Sample & $\begin{array}{c}\text { Young's modulus } \\
\text { (MPa) }\end{array}$ & Maximumstress (MPa) & Yield stress (MPa) & Breaking stress (MPa) & $\begin{array}{c}\text { Elongation atbreak } \\
(\%)\end{array}$ \\
\hline LDPE & $132.4 \pm 23.9$ & $12.2 \pm 1.6$ & $9.7 \pm 0.1$ & $11.7 \pm 1.3$ & $316.5 \pm 70.8$ \\
\hline $\mathrm{A}(75 / 25)_{\mathrm{N} 15}$ & $169.3 \pm 43.8$ & $4.0 \pm 0.2$ & $4.2 \pm 0.4$ & $2.4 \pm 1.6$ & $5.8 \pm 2.3$ \\
\hline $\mathrm{B}(90 / 10)_{\mathrm{N} 30}$ & $121.6 \pm 14.8$ & $11.9 \pm 0.2$ & $8.5 \pm 0.1$ & $11.4 \pm 0.5$ & $383.8 \pm 16.9$ \\
\hline$C(75 / 25)_{\mathrm{N} 30}$ & $92.0 \pm 11.1$ & $11.1 \pm 0.5$ & $8.1 \pm 0.2$ & $9.8 \pm 1.6$ & $318.5 \pm 75.5$ \\
\hline$D(50 / 50)_{\mathrm{N} 30}$ & $87.0 \pm 16.4$ & $7.7 \pm 0.2$ & $7.2 \pm 0.4$ & $7.3 \pm 0.8$ & $152.6 \pm 61.4$ \\
\hline$E(75 / 25)_{\mathrm{H} 15}$ & $166.6 \pm 32.0$ & $9.5 \pm 0.3$ & $9.3 \pm 0.6$ & $6.5 \pm 1.3$ & $20.7 \pm 4.4$ \\
\hline $\mathrm{F} 90 / 10)_{\mathrm{H} 30}$ & $123.4 \pm 23.9$ & $10.3 \pm 1.2$ & $8.7 \pm 0.1$ & $9.3 \pm 2.5$ & $276.6 \pm 125.1$ \\
\hline $\mathrm{G}(75 / 25)_{\mathrm{H} 30}$ & $109.0 \pm 18.2$ & $9.6 \pm 0.8$ & $7.9 \pm 0.2$ & $8.6 \pm 1.4$ & $258.7 \pm 58.6$ \\
\hline $\mathrm{H}(50 / 50)_{\mathrm{H} 30}$ & $89.0 \pm 2.9$ & $7.0 \pm 0.2$ & $6.4 \pm 0.3$ & $5.3 \pm 1.3$ & $88.2 \pm 20.2$ \\
\hline
\end{tabular}

LDPE: low-density polyethylene; Composites: $\mathrm{A}(75 / 25)_{\mathrm{N} 15}, \mathrm{~B}(90 / 10)_{\mathrm{N} 30}, \mathrm{C}(75 / 25)_{\mathrm{N} 30}, \mathrm{D}(50 / 50)_{\mathrm{N} 30}, \mathrm{E}(75 / 25)_{\mathrm{H} 15}, \mathrm{~F}(90 / 10)_{\mathrm{H} 30}, \mathrm{G}(75 / 25)_{\mathrm{H} 30}$, and $\mathrm{H}(50 / 50)_{\mathrm{H} 30}$; weight ratio LDPE/TPS letter A (75/25), B (90/10), C (75/25), D (50/50), E (75/25), F (90/10), G (75/25), and H (50/50); subscript N: native starch, subscript H: hydrolyzed starch; number 15 or 30 : percentage of glycerol in the TPS

Table 4: Thermal Properties of the LDPE/TPS Composites and Crystallinity of the Composites

\begin{tabular}{|c|c|c|c|c|}
\hline Sample & Tp( $\left.{ }^{\circ} \mathbf{C}\right)$ & Tc $\left({ }^{\circ} \mathbf{C}\right)$ & $\Delta \mathbf{H}_{\mathrm{f}}(\mathbf{J} / \mathbf{g})$ & Crystallinity $(\%)$ \\
\hline \hline LDPE & 110.71 & 96.87 & 106.7 & 36.07 \\
\hline $\mathrm{B}(90 / 10)_{\mathrm{N} 30}$ & 110.8 & 96.88 & 91.01 & 34.19 \\
\hline $\mathrm{C}(75 / 25)_{\mathrm{N} 30}$ & 110.58 & 97.07 & 85.22 & 38.41 \\
\hline $\mathrm{D}(50 / 50)_{\mathrm{N} 30}$ & 110.61 & 96.77 & 67.59 & 45.70 \\
\hline $\mathrm{E}(75 / 25)_{\mathrm{H} 15}$ & 110.45 & 97.48 & 101.0 & 35.53 \\
\hline $\mathrm{F}(90 / 10)_{\mathrm{H} 30}$ & 110.75 & 96.44 & 97.4 & 36.59 \\
\hline $\mathrm{G}(75 / 25)_{\mathrm{H} 30}$ & 111.17 & 96.64 & 81.53 & 36.75 \\
\hline $\mathrm{H}(50 / 50)_{\mathrm{H} 30}$ & 111.20 & 95.73 & 72.49 & 48.97 \\
\hline
\end{tabular}

LDPE: low-density polyethylene; Composites: $\mathrm{A}(75 / 25)_{\mathrm{N} 15}, \mathrm{~B}(90 / 10)_{\mathrm{N3}}, \mathrm{C}(75 / 25)_{\mathrm{N} 30}, \mathrm{D}(50 / 50)_{\mathrm{N} 30}, \mathrm{E}(75 / 25)_{\mathrm{H} 15}, \mathrm{~F}(90 / 10)_{\mathrm{H} 30}, \mathrm{G}(75 / 25)_{\mathrm{H} 30}$, and $\mathrm{H}(50 / 50)_{\mathrm{H} 30}$; weight ratio LDPE/TPS letter A (75/25), B (90/10), C (75/25), D (50/50), E (75/25), F (90/10), G (75/25), and H (50/50); subscript N: native starch; subscript H: hydrolyzed starch; number 15 or 30: percentage of glycerol in the TPS; $T_{p}$ : Peak temperature of fusion; $T_{c}$ : Crystallization Temperature; $\Delta \mathrm{H}_{\mathrm{f}}$ : Fusion enthalpy of the composites.

that exhibit the lowest values for the modulus. This finding indicates that the morphology developed in the composites and that the shape and size of the TPS particles in the matrix have a greater effect on the physicochemical properties of the obtained composites.

\section{FRACTURE MORPHOLOGY OF THE LDPE/TPS COMPOSITES ANALYZED VIA SCANNING ELECTRON MICROSCOPY (SEM)}

The morphology of the LDPE and the LDPE/TPS composites originating from native starch with a $30 \%$ glycerol concentration are shown in the micrographs in Figure 6. The composites were freeze-fractured, and the samples were analyzed at the fractures. The matrix exhibits ductile fracturing, given that the surface of the matrix exhibits areas where the material was deformed during the fracture. The composite $\mathrm{B}(90 / 10)_{\mathrm{N} 30}$ exhibits a different fracture morphology from that of the matrix, given that areas of greater relief and a more granular texture can be observed. Darker areas with hemispherical shapes can also be observed, which is typical forthe presence of thermoplastic starch. The size distribution of the starch particles ranges from 0.3 to $1 \mu \mathrm{m}$. At $25 \%$ by weightof TPS in the LDPE matrix, an increase in the size of the TPS particles to between 0.5 and $2.5 \mu \mathrm{m}$ is observed. In the case of the composites with $50 \%$ by weight of TPS, although there is no longer any significant increase in the size of the particles, the number of larger particles increases (0.62.7 $\mu \mathrm{m})$. This finding may be due to the phenomenon of coalescence between particles during the preparation of the composites [11].

The glycerol content of the TPS also affects the morphology of the composites. Figure 7 shows the micrographs of the LDPE/TPS (75/25) composites obtained from hydrolyzed starch, with glycerol 

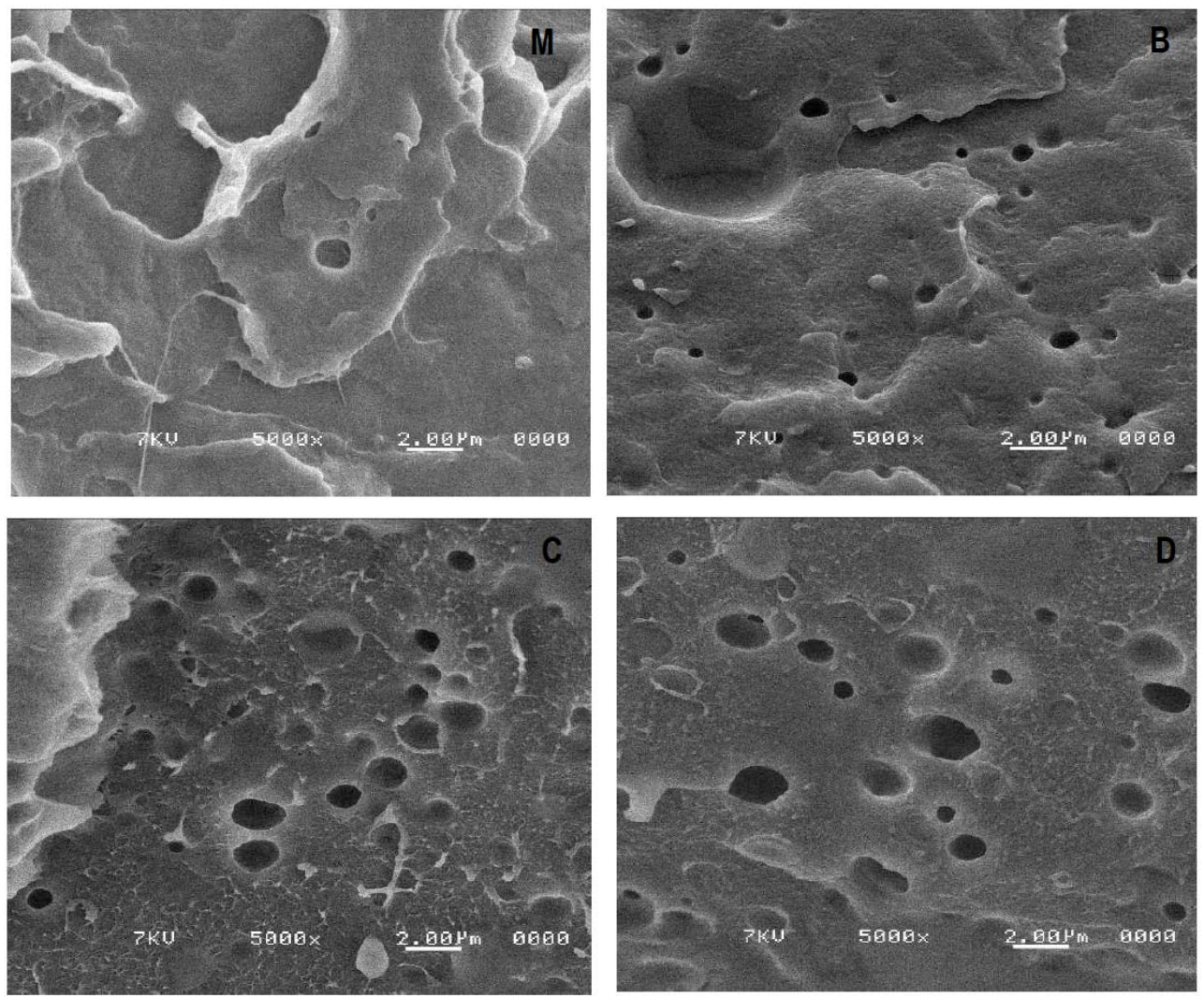

Figure 6: Morphology of the fracture of LDPE (M) and the LDPE/TPS N30 composites at different concentration of TPS originating from native starch and 30\% glycerol. B (90:10) N30, C (75:25) N30, and D (50:50) N30, observed at an amplification of $5000 \mathrm{X}$
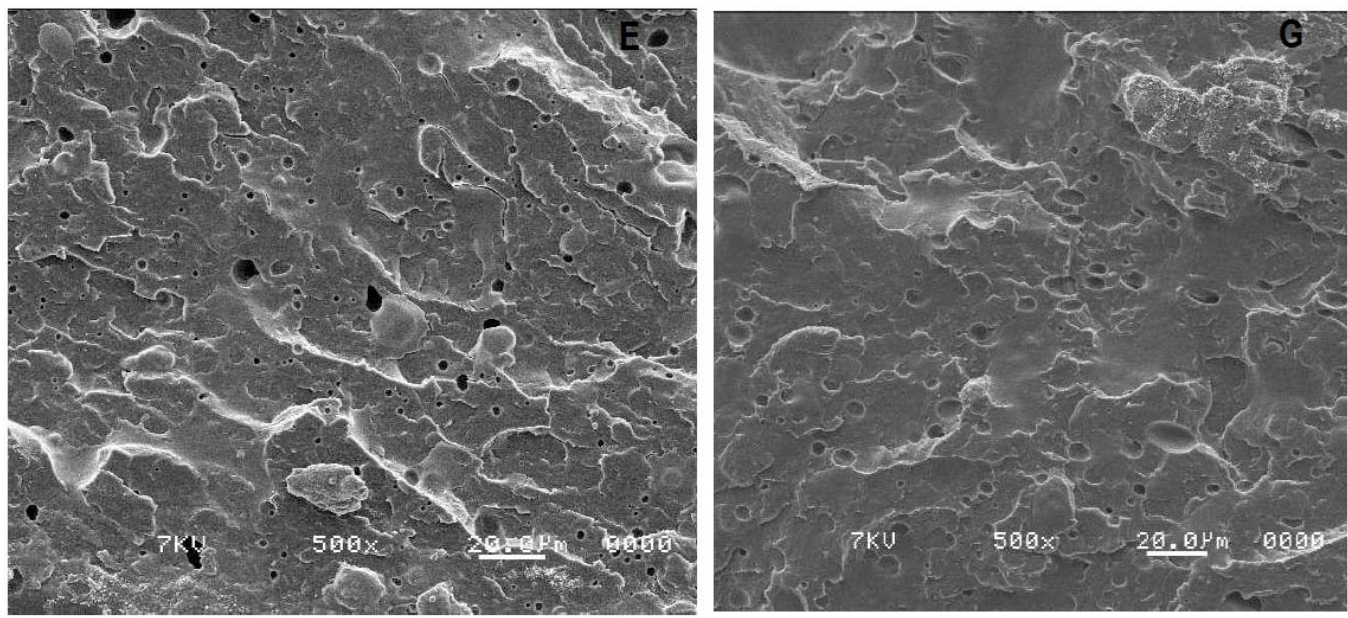

Figure 7: The effect of glycerol on the morphology of the LDPE/TPS $(75: 25)_{\mathrm{H}}$ composites with a glycerol concentration of $15 \%$. $\mathrm{E}(75: 25)_{\mathrm{H} 15}$ and $30 \% \mathrm{G}(75: 25){ }_{\mathrm{H} 30}$ freeze-fractured, observed at an amplification of $500 \mathrm{X}$.

concentrations of 15 and $30 \%$, respectively. With $15 \%$ glycerol in the TPS, the morphology of the fracture exhibits a fragile structure, and some cracks in the surface can even be observed. The average size of the TPS particles is $2.84 \mu \mathrm{m}$, and they have a near spherical morphology. In the case of the composites containing TPS with a $30 \%$ glycerol content, the type of fracturing is less fragile, and the average size of the particlesis $5.14 \mu \mathrm{m}$. Moreover, a percentage of these particles have an ellipsoidal morphology, which can be attributed to fact that the viscosity of the TPS is lower and that it can therefore be more easily deformed during the preparation of the composites. The difference in the size of the particles and the type of morphology of the composites containing 15\% TPS explain the large increase in Young's modulus, which is 


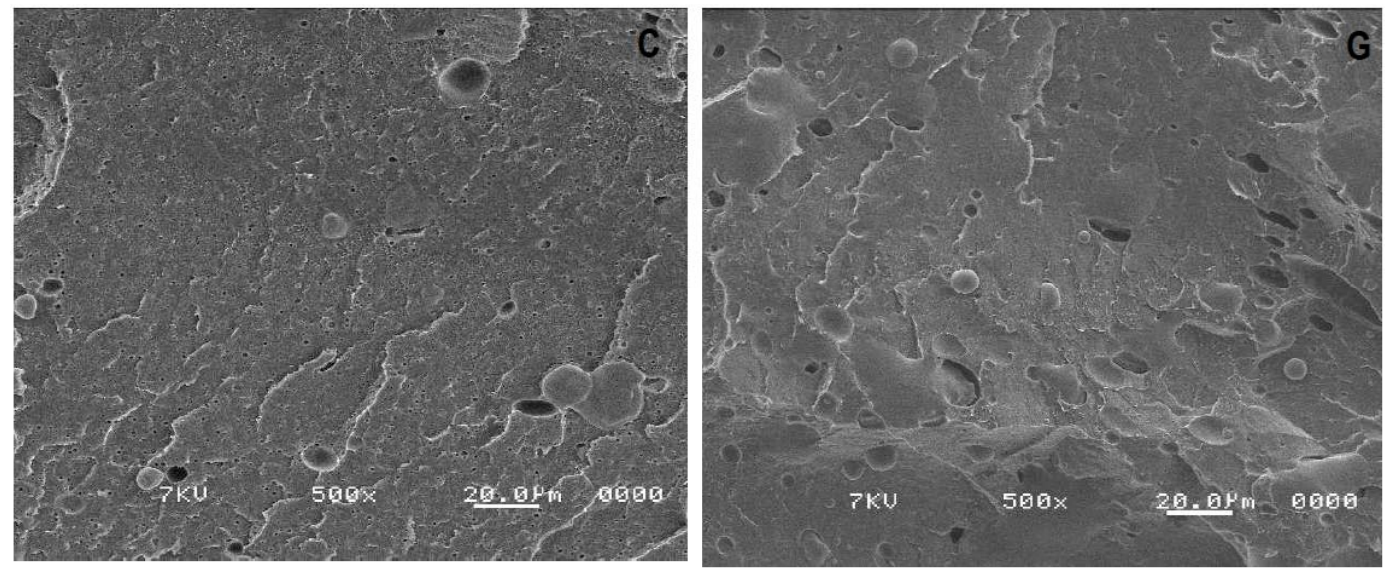

Figure 8: The effect of the type of TPS on the morphology of the composites C) native LDPE/TPS (75/25) $)_{\mathrm{N} 30}$ and G) hydrolyzed LDPE/TPS (75:25) нзо . The TPS contains 30\% glycerol.

even higher than that of the matrix, in addition to the reduced deformation of the composites.

The type of TPS added to the polyethylene matrix also has a significant effect on the morphology of the composites. In Figure 8, a few of the micrographs of the LDPE/TPS (75/25) composites with TPS containing $30 \%$ glycerol are shown. In can be observed that for the composites that contain TPS obtained from native starch, the particles are homogeneously distributed in the matrix and have an average size of $1.04 \mu \mathrm{m}$, whereas the composites with hydrolyzed starch contain particles with an average size of $5.14 \mu \mathrm{m}$. Moreover, a fraction of the particles that exhibit fibrillar morphology can be observed. These particles may be generated as a result of the reduction in the viscosity of the TPS as a product of hydrolysis. The significant increase in the size of the particles of the composites that contain hydrolyzed TPS may explain why both the maximum stress and the deformation of the composites are lower in comparison to the composites that contain native TPS.

\section{CONCLUSIONS}

It is found that composites of LDPE containing hydrolyzed TPS exhibits some differences in terms of physicochemical properties that composite containing native TPS, the Young's modulus of the composites with hydrolyzed TPS is greater than that of the composites that contain native TPS, although both maximum tensile strength and deformation are lower in the composites that contain hydrolyzed TPS. It is also observed that the addition of TPS causes an increase in the degree of crystallinity of LDPE. Similarly, hydrolyzed TPS causes a greater increase in crystallinity than native TPS. The size of the hydrolyzed
TPS particles in the matrix increases significantly compared to composites with native TPS. This finding may be useful in a range of applications, given that these composites may be more susceptible to biodegradation.

\section{ACKNOWLEDGEMENTS}

We would like to express our gratitude to the National Council for Science and Technology (Consejo Nacional de Ciencia y Tecnología - CONACYT) for presenting the grant (number 325990) to grant holder Perla M. Trejo Ramírez (record number 221083). Similarly, we thank the Coah-2013-C24-203199 project for its financial support during the completion of the present study. In addition, we would like to thank Francisco Zendejo, Rodrigo Cedillo, José Luis Saucedo Morales, Carlos A. Gallardo Vega, Guadalupe Méndez, and Myriam Lozano for their technical support and analysis of the samples.

\section{REFERENCES}

[1] Hood LF. Current concepts of starch structure. In Food Carbohydrates, Lineback, DR, Inglett GE,editores. Avi Publishing Co. Inc, Westport, C.T. EUA. 1982; pp. 217-36.

[2] Belitz HD, Grosch W, Schieberle P. Food Chemistry. 4ㄹed Springer -Verlag Berlin Heidelberg 2009; pp. 249-60.

[3] Gao J, Vasanthan T, Hoover R, Li J. Structural modification of waxy, regular, and high-amylose maize and hulless barley starches on partial acid hydrolysis and their impact on physicochemical properties and chemical modification. Starch/Stärke 2012; 64: 313-25. http://dx.doi.org/10.1002/star.201100128

[4] Huber KC, BeMiller JN. Location of sites of reaction within starch granules. Cereal Chem 2001; 78: 173-80. http://dx.doi.org/10.1094/CCHEM.2001.78.2.173

[5] Avérous L, Moro L, Dole P, Fringant C. Properties of thermoplastic blends: starch-polycaprolactone. Polymer 2000; 41: 4157-67. http://dx.doi.org/10.1016/S0032-3861(99)00636-9 
[6] Avérous L, Faunonnier N, Moro L, Fringant C. Blends of thermoplastic starch and polyesteramide: Processing and properties. J Appl Polym Sci 2000; 76: 1117-28.

http://dx.doi.org/10.1002/(SICI)1097-

4628(20000516)76:7<1117::AID-APP16>3.0.CO;2-W

[7] Walia PS, Lawton JW, Shogren RL. Mechanical properties of thermoplastic starch/poly (hydroxy ester ether) blends: effect of moisture during and after processing. J Appl Polym Sci 2002; 84: 121-31.

http://dx.doi.org/10.1002/app.10271

[8] Kaseem M, Hamad K, Deri F. Thermoplastic starch blends: a review of recent works. Polym Sci Series A 2012; 54: 165-76. http://dx.doi.org/10.1134/S0965545X1202006X

[9] Huneault MA, Li H. Preparation and properties of extruded thermoplastic starch/polymer blends. J Appl Polym Sci 2012; 126: E96-108.

http://dx.doi.org/10.1002/app.36724

[10] Rodríguez González FJ, Ramsay BA, Favis BD. High performance LDPE/thermoplastic starch blends: a sustainable alternative to pure polyethylene. Polymer 2003; 44: 1517-26.

http://dx.doi.org/10.1016/S0032-3861(02)00907-2

[11] Méndez Hernández ML, Tena Salcido CS, Sandoval Arellano Z, González Cantú MC, Mondragón M, Rodríguez González FJ. The effect of thermoplastic starch on the properties of HDPE/TPS blends during UV-accelerated aging. Polym Bull 2011; 67: 903-14.

http://dx.doi.org/10.1007/s00289-011-0501-4

[12] Favis BD, Rodríguez González FJ, Ramsay BA. Polymer Compositions Containing Thermoplastic Starch.United Statespatent US 66056572003 Aug.

[13] Kiatkamjornwonga S, Thakeowb P, Sonsukc M. Chemical modification of cassava starch for degradable polyethylene sheets. Polym Degrad Stab 2001; 73: 363-75. http://dx.doi.org/10.1016/S0141-3910(01)00100-8

[14] Ma XF, Yu JG. The plasticizers containing amide groups for thermoplastic starch. Carbohydr Polym Deg Stab 2004; 57: 197- 203.

http://dx.doi.org/10.1016/j.carbpol.2004.04.012

[15] Thomas DJ, Atwell WA. Starches practical guides for the food industry, St. Paul, Minnesota, USA 1997.

[16] Hebeish A, El-Thalouth I, Refai R, Dokki AR. Synthesis and characterization of hypochlorite oxidized starches. Starch/Stärke 1989; 41: 293-8. http://dx.doi.org/10.1002/star.19890410804

[17] Bao M, Ersun A, Kavanç G. The evaluation of food hygiene knowledge, attitudes and practice of food handlers in food businesses in Turkey. Food Control 2006; 17: 317-22.

http://dx.doi.org/10.1016/j.foodcont.2004.11.006
[18] Day JT, Malzahn RC, Morehouse AL. Hydrolysis of starch. United Statespatent US3663369 A.1972 May.

[19] Zambrano FM, Camargo CR. Substituto de gordura: efeito da concentração de ácido e do tempo de hidrólise do amido de mandioca em algumas características dos hidrolisados. Braz J Food Technol 1999; 2: 155-9.

[20] Mark JE. Physical Properties of Polymer Handbook. AIP Press: New York 1996.

[21] Almeida MR, Alves RS, Nascimbem LBLR, Stephani R, Poppi RJ, de Oliveira LFC. Determination of amylose content in starch using Raman spectroscopy and multivariate calibration analysis. Anal Bioanal Chem 2010; 397: 2693701.

http://dx.doi.org/10.1007/s00216-010-3566-2

[22] Tu AT, Lee J, Milanovich FP. Laser-Raman spectroscopic study of cyclohexaamylose and related compounds; spectral analysis and structural implications. Carbohydr Res 1979; 76: $239-44$ http://dx.doi.org/10.1016/0008-6215(79)80023-3

[23] Sekkal M, Dincq V, Legrand P, Huvenne JPJ. Investigation of the glycosidic linkages in several oligosaccharides using FTIR and FT-Raman spectroscopies. Mol Struct 1995; 349: 349-52.

http://dx.doi.org/10.1016/0022-2860(95)08781-P

[24] Phillips DL, Xing J, Liu H, Pan DH, Corke H. Potential use of Raman spectroscopy for determination of amylose content in maize starch. Cereal Chem 1999; 76: 821-3. http://dx.doi.org/10.1094/CCHEM.1999.76.5.821

[25] Mortazavi S, Ghasemi I, Oromiehie A. Effect of phase inversion on the physical and mechanical properties of low density polyethylene/thermoplastic starch. Polym Test 2013; 32: 482-91. http://dx.doi.org/10.1016/j.polymertesting.2013.01.004

[26] Park JW, Kim GH, Moon JB. Effects of a compatibilizer on the tensile properties of low-density polyethylene/modified starch blends. J Environ Sci Int 2013; 22: 1287-94. http://dx.doi.org/10.5322/JESI.2013.22.10.1287

[27] Willett JL. Mechanical properties of LDPE/Granular starch composites. J Appl Polym Sci 1994; 54: 1685-95. http://dx.doi.org/10.1002/app.1994.070541112

[28] Ratto JA, Stenhouse PJ, Auerbach M, Mitchell J, Farrel R Procesing performance and biodegradability of a thermoplastic aliphatic polyester/starch system. Polymer 1999; 40: 6777-88. http://dx.doi.org/10.1016/S0032-3861(99)00014-2

\section{DOI: http://dx.doi.org/10.6000/1929-5995.2015.04.04.4}

(c) 2015 Trejo-Ramírez et al.; Licensee Lifescience Global.

This is an open access article licensed under the terms of the Creative Commons Attribution Non-Commercial License (http://creativecommons.org/licenses/by-nc/3.0/) which permits unrestricted, non-commercial use, distribution and reproduction in any medium, provided the work is properly cited. 